\title{
Beads, Pendants, and the Like
}

\author{
Jocelyn Simlick, Andrea Miloslavic, and Vanessa Davies
}

Explanation of the bead and pendant catalog:

The finds' description of context below is taken from the Catalogue of Tombs. The photograph numbers reflect the original excavator's numbering system.

Description of objects and count of objects (exact or approximate).

Typology: Made according to descriptions in Beck (2006, Part II), and Harrell (2017, Table 2), as well as numbers from Xia Nai's (2014) bead corpus. Following Reisner $(1932,149)$, the distinction between ring and disk beads is that rings are 2-4 $\mathrm{mm}$ in diameter, and disks are $4-10 \mathrm{~mm}$ in diameter.

Material: Agate, amethyst, carnelian, coral, Egyptian blue, faience, feldspar, glass, gold, metal, mud, onyx, ostrich eggshell, quartz, seed, shell, steatite, travertine.

Color: Red, orange, yellow, gold (yellow, rose, brown), green, blue, purple, brown, black, white. Measurements: Length, diameter, weight, and others as relevant. Aperture measurements were collected only for unstrung objects.

Production: Determined according to Xia (2014). For the abbreviations омма/в (Ordinary Modeling Method A/B) and SMмc/D (Special Modeling Method C/D), see Xia $(2014,38)$. Production method was not noted if the stone was simply carved.

Edge/no edge: According to Beck $(2006,3)$. Aperture: Apex/end, according to Beck (2006, 2).

Perforation: According to Beck (2006, Part III) and Harrell (2017, Table 2). Recorded only for hard and soft stones and shells. Not relevant to faience, glass, paste, and metal objects.

Striations: Longitudinal/rotary/random, according to Xia (2014, Section 7.1). Relevant only for hard stone. Decoration: According to descriptions in Beck (2006, Part VI) and Xia (2014).

Glaze: Mostly worn/mostly not worn. Polish: High/moderate/dull/rough.

N 2010 Map D4; photo B 7129

In large chamber, with a number of $\mathrm{A}$ and $\mathrm{M}$ pots, a few dark and light glass disc beads (PAHMA 6-14302). 
(1) PAHмA 6-14302 Loose beads

Exactly 41 beads.

Typology: Disc, barrel—PN2g (40); spacer-PD18e (shell, 1). Material: Faience. Color: Green (19); black (22).

Length: $0.75^{-2.75} \mathrm{~mm}$. Diameter: $3^{6} 65^{-6.85} \mathrm{~mm}$. Aperture: $0.85^{-1.37} \mathrm{~mm}$. Weight: $1.2 \mathrm{~g}$.

Production: OMMA (faience). No edge.

Aperture: End.

Glaze: Mostly not worn.

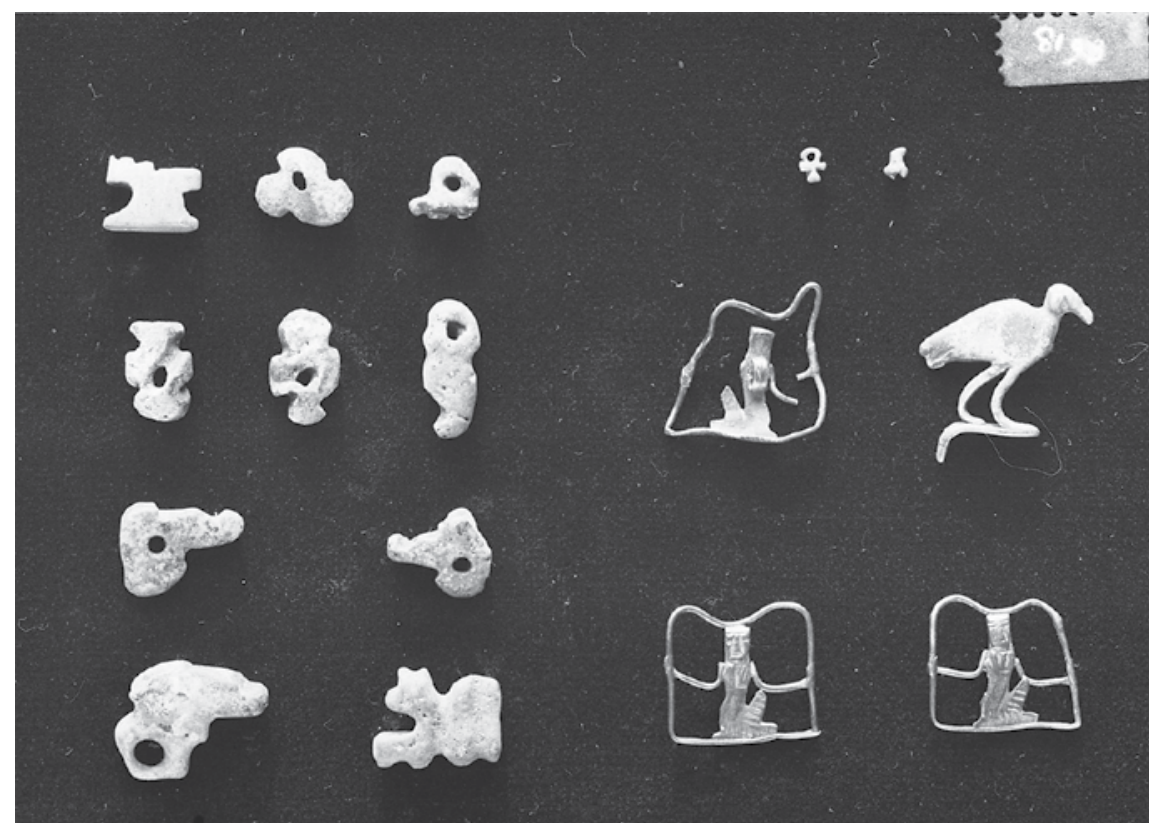

FIGURE 7.1 (Left): 6-14462 (N 2235); (Right): 6-12515 (N 2834), 6-14304a-c (N 2016), 6-14547 (N 2618) (NED-C-8193)

N 2016 Map D4; photos A 8267, B 8251, C 8193, C 8464 (Figs. 7.1, 7.3)

Burial utterly plundered. Scattered three gold brooches (PAнMA 6-14304a-c) and stone beads, cylindrical (PAHMA 6-14303). In filling at top of pit, small glass bottle, piece of a large dish with Coptic inscription scratched on it, and broken pottery.

(1) PAHмA 6-14303 Loose beads and pendant

Exactly 13 objects (12 beads and 1 pendant). 
Typology: Cylindrical, long-L16q (glazed steatite, 4); barrel, short- $\mathrm{PN}_{17} \mathrm{~h}$ (faience, 2); barrel, short- $\mathrm{H}_{15} \mathrm{~m}$ (carnelian, 1); figural —2 lions, 1 double lion head, 1 Tawaret, 1 paddle (?), 1 pendant (Ihy? Khonsu? Shed?) (faience, 6).

Material: Carnelian (1), steatite (4), faience (8).

Color: Orange (carnelian); green (faience); light brown (faience); brown (faience, glazed steatite). Length of objects: $1.84-28.88 \mathrm{~mm}$.

Diameter of objects: $3.63-6.84 \mathrm{~mm}$. Aperture of objects: $0.14-2.37 \mathrm{~mm}$. Weight: $0.2-2.3 \mathrm{~g}$.

Production: оммв (faience). No edge.

Aperture: End.

Perforation: Double cone (carnelian); plain (steatite). Striations: Random (carnelian); longitudinal (steatite). Glaze: Mostly not worn (steatite); mostly worn (faience). Polish: Moderate (carnelian).

For parallels, see the following: Lion: Brooklyn Museum 16.366; Petrie 1914, pl. 38:219; Reisner 1923, 128, pl. 44,2,20-21; Brunton 1948, pl. 31:26. Double lion head: Brunton 1928, pl. 95:17D6. Tawaret: PAH MA 6-14306 (N 2021); Petrie 1914, pl. 40:236; Brunton 1948, pl. 31:37. Child amulet, Petrie 1914, pl. 26:145.

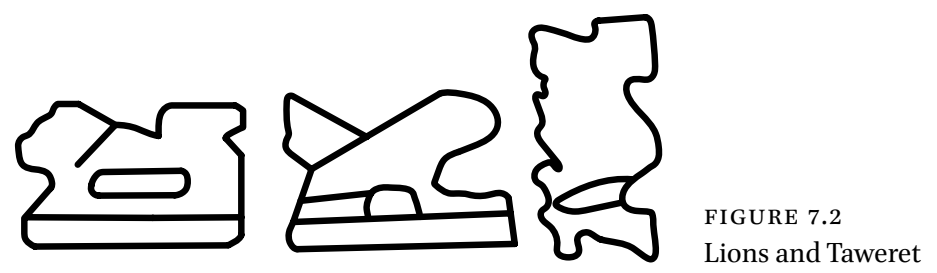

(2) PAH MA 6-14304a-c Loose pendants

Exactly 3 pendants.

Typology: Figural (heh hieroglyphs). Material: Gold.

Color: Yellow gold. Length: 1.6o-1.85 mm. Diameter: 3.35-3.6o mm.

Aperture: $1.60-1.82 \mathrm{~mm}$.

Head to feet: $14.40 \mathrm{~mm}(\mathrm{a}), 14.10 \mathrm{~mm}(\mathrm{~b}), 15.75 \mathrm{~mm}$ (c). Arm to arm: $15.25 \mathrm{~mm}$ (a), $15.13 \mathrm{~mm}$ (b), $16.25 \mathrm{~mm}$ (c). Weight: $2.5 \mathrm{~g}$.

Production: Molding (metal body); soldering (arms and reeds: wire soldered together and soldered to body). Aperture: End.

Perforation: Single plain.

Decoration: Metal has carved details (face and garment).

For parallels, see Brooklyn Museum 13.1035, 13.1036, 13.1037; MFA 13.3772; MMA 04.16.6, 04.16.10, 04.16.11, 04.16.13, 04.16.14, 11.215.206; BM ЕA 65331, 66662; 
UC 18025; EG 5072; Petrie 1914, pls. 4:59a-d, 46:59e; Andrews 1997, fig. 4e; Brunton 1948, pl. 32:81.

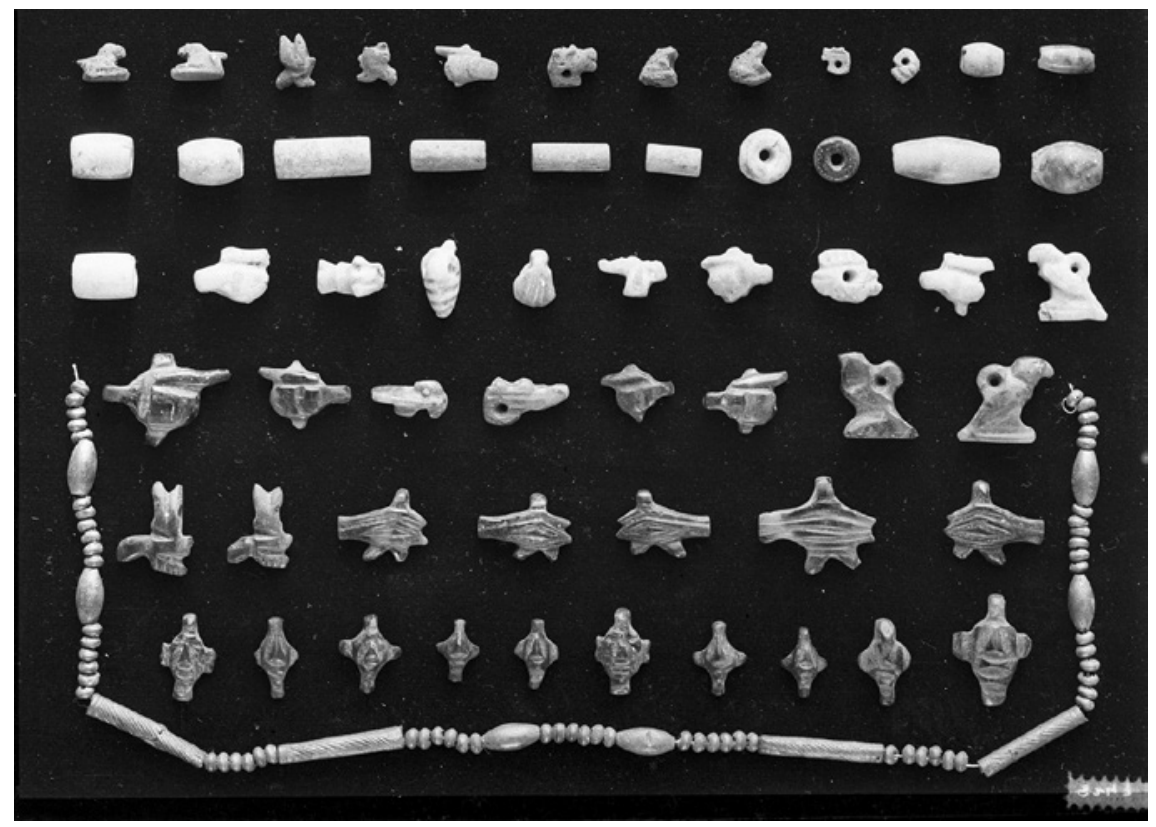

FIGURE 7.3 (Rows 1-3): 6-14307; (Rows 4-6): 6-14306; (bottom): 6-14305 (N 2021) (NED-B-8247)

N 2021 Map N/A [ed.: This record is Reisner's note of preliminary work that was done in 1901. But according to Hearst Museum records, the objects below were found in N 2021.] photos A 4943, B 8247, C 9628, C 9629 (Fig. 7.3)

Empty pits. Rectangular cloth. Shells, etc.

(1) PAHMA 6-14305 Bracelet

Exactly 74 beads.

Typology: Barrel, short-Mgf (6); cylindrical, short-Mi2p (4); ring, biconical-M2c (64). Material: Faience covered in gold.

Color: Brown gold.

Length of strand: $13.1 \mathrm{~mm}$. Diameter of strand: $8.5 \mathrm{~mm}$. Length of beads: 1.34-19.93 mm. Diameter of beads: 2.75-4.16 mm. Weight: $2.2 \mathrm{~g}$.

Production: Could not determine (faience); plating on core (metal, with faience as the core). Edge (ring beads), no edge (cylindrical and barrel beads). Aperture: End. 
Decoration: Metal cylindrical beads decorated by the burnished on core method with 2 lines on either end of the bead that run perpendicular to the length of the bead (the type of decoration that Beck (p. 66) refers to as a "zone bead") and a spiral design in the center of the bead.

Polish: Moderate.

(2) PAHMA 6-14306 Bracelet

Exactly 25 pendants.

Typology: Figural—10 face (full-frontal); 4 fist (2 left, 2 right); 5 eye of Horus; 1 Tawaret; 1 lion head; 2 bee; 2 falcon.

Material: Carnelian. Color: Red, orange.

Length of pendants: $1.90-4.77 \mathrm{~mm}$. Diameter of pendants: $3.5^{0}-5.58 \mathrm{~mm}$. Weight: 14.o g.

No edge. Aperture: End. Perforation: Double cone. Striations: Random. Decoration: Carved. Polish: High.

For parallels, see the following: Head: Petrie 1914, pl. 1:2a-f; BM EA 30416; Brunton 1948, pl. 31:12-13; fist: Petrie 1914, pl. 1:12a-c; eye of Horus: Petrie 1914, pl. 12:94. Tawaret: PAH MA 6-143O3 (N 2016); Petrie 1914, pl. 40:236; Brunton 1948, pl. 31:37. Lion head: UC 18117; BM EA 57774i (Andrews 1997, fig. 5e); UC 38551; Petrie 1914, pl. 2:22f; Brunton 1928, pl. 95:16, 1948, pl. 31:33-34; PAH MA 6-10067 from N 570. Bee: Petrie 1914, pl. 4:47; MMA 59.103.18, 59.103.19, 59.103.21. Falcon: Petrie 1914, pl. 41:245af; Reisner 1907, pl. 25:12516, 12517, 12526; MMA 26.7.895; BM EA 58319 .

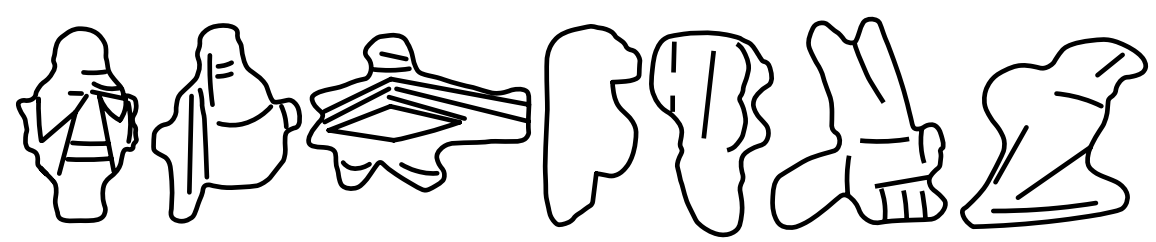

FIGURE 7.4 Face (frontal), fist, eye of Horus, Tawaret, lion head, bee, falcon

(3) PAHмA 6-14307 Bracelet, loose beads, and pendants

Exactly 31 objects ( 20 beads and 11 pendants).

Typology: Barrel, long- $\mathrm{PN} 18 \mathrm{~h}$ (faience, 1); barrel, short- $\mathrm{H} 15 \mathrm{r}$ (carnelian, agate, 7); cylindrical, long-PN22r (faience, 1); cylindrical, long-S18i (glazed steatite, 4); disc, cylindrical- $\mathrm{H}_{2} \mathrm{n}$ (carnelian, 1); disc, biconical- $\mathrm{H}_{5} \mathrm{l}$ (green feldspar, 1); cylindrical, short-H22b (green feldspar, 2); figural —2 lions, 3 lion heads, 1 broken unknown (faience, 5); 2 shells, 1 falcon, 1 fish, 2 fists, 2 broken unknown (green feldspar, 9).

Material: Faience (7), carnelian (2), steatite (6), green feldspar (12), agate (4). 
Color: Blue, brown (faience); orange, white (carnelian, agate); green (feldspar); light brown (glazed steatite). Length of objects: 1.68-16.52 mm.

Diameter of objects: 4.09-8.13 mm. Weight: $13.1 \mathrm{~g}$.

Production: омма (1 faience), оммв (1 faience); molded (figured faience); carved (figured hard stone).

Edge (some); no edge (some). Aperture: End.

Perforation type (unstrung beads only): Biconical (agate); single plain (feldspar). Striations: Rotary (barrel, biconic disc: feldspar, carnelian, agate); longitudinal (cylindrical: feldspar, steatite); random: (cylindrical: carnelian disc). 1 green feldspar has a "basket"-patterned striae.

Glaze: 1 not worn, remainder mostly worn (faience); mostly worn (steatite). Polish: High (agate); moderate (carnelian); dull to moderate (feldspar).

For parallels, see the following: Lion: Brooklyn Museum 16.366; Petrie 1914, pl. 38:219; Reisner 1923, 128, pl. 44,2,20-21. Shells: Petrie 1914, pl. 14:109,111; MMA 10.130.2436; вм EA 58574. Falcon: Petrie 1914, pl. 41:245af; Reisner 1907, pl. 25:12516, 12517, 12526; M MA 26.7.895; BM EA 58319. Lion head: UC 18117; BM EA 57774i (Andrews 1997, fig. 5e); UC 38551; Petrie 1914, pl. 2:22f; Brunton 1928, pl. 95:16, 1948, pl. 31:33-34; PAH MA 6-10067 from N 570. Fist: Petrie 1914, pl. 1:12; BM EA 57832 .
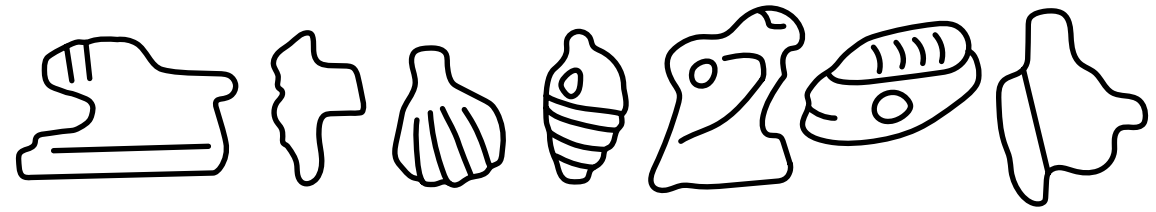

FIGURE 7.5 Lion, lion head, shells, falcon, fish, fist

N 2031 Map E3; photo C 983, C 7178, C 7179, C 8199

These objects also found with a stela face down and on top of coffin. Coffin had angled corner. By neck, quantity of gold foil (PAHMA 6-14310) had been upon something made of wood. Traces of coffin under feet, and apparently under coffin, few bones of a calf or some other small animal. Scattered about upper part of body, number of small glaze disc beads, green stone amulet, with gold lining inside, carnelian, white stone, green stone, carnelian, glaze, soapstone?, blue stone (PAHMA 6-14317). Under coffin, pottery. Quantity of A, $M$ with white inside rim (PAHMA 6-14313, PAHMA 6-14318, PAHMA 6-14321), and (PAHMA 6-14311, PAHMA 6-14314, PAHMA 6-14315, PAHMA 6-14319, PAHMA 6-14320). Slight groove and knife mark (PAHMA 6-14312). Broken clay jar stopper. Also ordinary stoppers. 
(1) PAHMA 6-14310 Flakes of gold foil

Color: Yellow and red.

Too fragile to be measured.

(2) PAH MA 6-14317 Length of necklace (not a loop) and loose beads Approximately 1065 beads (1035 strung and 30 loose).

Typology: Barrel, short-H15n (carnelian, 1); barrel, long-L12k (steatite, 1); cylindrical, short-PN22c (faience, 3), H21h (carnelian, 1); cylindrical, longPN22r (faience, 1), H15i (onyx, 1); disc, barrel—- 55 j (carnelian, 2); ring, barrel$\mathrm{PN} 2$ (faience, approx. 1035 in necklace, 15 loose); ring, cylindrical-H6f (feldspar, 1), M3b (metal, 2); 2 figural-tilapia Nilotica fish (blue, 1), 1 hippopotamus(?) head (in profile; made to hang upside down; unknown material, 1). Material: Faience (approx. 1035 in necklace and 20 loose beads), carnelian (4), steatite (1), green feldspar (1), onyx (1), metal (2), Egyptian blue (1), unknown (1). Color: Orange (carnelian); green (feldspar, faience, unknown); blue (Egyptian blue); brown (metal, steatite, onyx).

Length of strand: $69 \mathrm{~cm}$.

Length of beads: $0.61-7.56 \mathrm{~mm}$. Diameter of beads: 1.69-4.85 mm. Aperture of loose beads: $0.53^{-1.60} \mathrm{~mm}$. Weight: $6.8 \mathrm{~g}$.

Production: RsmB (joined) (metal).

Edge (2 carnelian disc beads); no edge (all the rest). Aperture: End.

Perforation: Double cone (2 carnelian barrel disc, 1 carnelian barrel short); single plain (steatite, onyx, feldspar, carnelian cylinder).

Striations: Longitudinal (steatite).

Glaze: Mostly not worn (faience, steatite).

Polish: High (carnelian, onyx); moderate (carnelian, green feldspar).

For parallels, see the following: carnelian disc bead with edge, Reisner 1908, 85, 118. Fish: Petrie 1914, pl. 43:257a, 50:257; MMA 22.1.1944y, 22.1.1944z; Germond 2005, fig. 28.

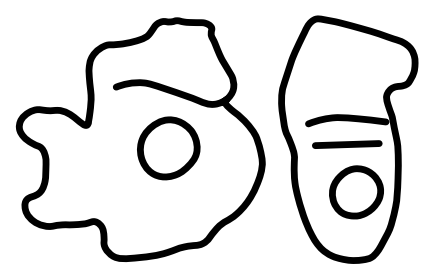

FIGURE 7.6

Fish, hippopotamus(?) head

N 2042 Map B5; type vi c; photo C 7496, C 7498, C 7499, C 750o, C 7501, C 7502, $\mathrm{C}_{75} \mathrm{O} 3, \mathrm{C} 75 \mathrm{O} 4, \mathrm{C}_{75} \mathrm{O} 5, \mathrm{~A} 8265$ 
Round pelvis girdle of 15 strings of shell and black and green glaze beads (PAHMA 6-14332, PAHMA 6-14341, PAHMA 6-14343). Order from below: 1. all green, 2. all white, 3. black and white, 4 . black and white, 5 . all white, 6 . black and white, 7. black and white with few green, 8 . black and white, 10. white, 11. black and white, 12. black and white. Strings were under both arms. All upper part broken owing to falling apart of pelvis. Underneath, 8 strings in position. Strings join at intervals of about an inch. Both upper and lower layers of girdle were underneath pelvis. Possibly it was a double girdle as all upper part was disturbed. There were extra strings; 1 of tiny glaze disc, and 1 of carnelian disc (PAH MA 6-14333, PAHMA 6-14335) and tiny glaze amulets (PAHMA 6-14334, PAHMA 6-14340).

These objects were found with 2 alabaster objects (PAHMA 6-14342), granite paint grinder, shell wand for kohl, bone kohl spatula, and alabaster kohl pot (PAHMA 6-14331). In filling, fossil, bronze needle. 2 alabaster at 8194 1/4 (PAHMA 6-14331) and 8194 2/4 (PAHMA 6-14342). [ed.: According to Hearst Museum records, PAHMA 6-14336 (loose beads), PAHMA 6-14337 (travertine bead), PAнмA 6-14338 (carnelian ball beads), PAHMA 6-14339 (faience ball beads) are from this tomb.]

(1) PAHMA 6-14332 Loose beads

Approximately 200 beads.

Typology: Disc, barrel—PN2g (faience, approx. 112); ring, barrel—H2e (carnelian, 1), R52f (ostrich eggshell, approx. 67); ring, cylindrical—PN2b (faience, approx. 20).

Material: Faience (approx. 132), carnelian (1), ostrich eggshell (approx. 67). Color: Red (carnelian); black (faience); white (ostrich eggshell).

Length: $0.90-1.53 \mathrm{~mm}$. Diameter: $2.36-4.39 \mathrm{~mm}$. Aperture: $0.50-1.50 \mathrm{~mm}$. Weight: 9.o g.

Production: om ma (faience). No edge.

Aperture: End.

Perforation: Double cone, single plain (ostrich eggshell). Striations: Rotary. Glaze: Mostly worn (faience).

These beads probably originally were part of the girdle (PAHMA 6-14343).

(2) PAHMA 6-14333 Length of necklace (not a loop)

Exactly 387 beads.

Typology: Ring, barrel—H6e (faience); ring, cylindrical—PN2c (carnelian). Material: Faience (222); carnelian (165).

Color: Red, orange (carnelian); black (faience). Length of strand: $35 \mathrm{~cm}$. 
Length of beads: $0.70-0.95 \mathrm{~mm}$. Diameter of beads: 2.35-2.6o mm. Weight: $2.3 \mathrm{~g}$. Production: omma (faience). No edge.

Aperture: End.

Perforation: Single plain (carnelian). Striations: Random.

Glaze: Mostly worn (faience). Polish: Moderate (carnelian).

(3) PAнмa 6-14334 Pendant

Exactly 1 pendant now in 4 pieces.

Typology: Figural-human figure (no head) with false beard.

Material: Faience. Color: Brown. Length: 3.46 mm. Diameter: $5.84 \mathrm{~mm}$.

Weight: Did not register. No edge.

Aperture: Unknown because pendant is broken. Production: Molding.

Glaze: Mostly worn.

(4) PAH MA 6-14335 Loose beads and pendants

Exactly 15 objects (12 beads and 3 pendants).

Typology: Barrel, long-PN18l (faience, 1); cylindrical, long-PD63f (faience, 2); ring, barrel- $\mathrm{H}_{2 \mathrm{e}}$ (carnelian, 8); ring, cylindrical- $\mathrm{R}_{52 \mathrm{f}}$ (shell, 1); figuraldouble humped (sema tawy?), human(?) figure, broken (faience, 3).

Material: Faience (6), carnelian (8), shell (1).

Color: Red (carnelian), orange (carnelian), brown (faience), white (shell). Length: $0.80-21.03 \mathrm{~mm}$.

Diameter: 2.32-7.90 mm. Aperture: 0.55-1.93 mm. Weight: $1.3 \mathrm{~g}$.

Production: OMMA, molding (faience). No edge.

Aperture: End.

Perforation: Double cone (carnelian), single plain (shell). Striations: Objects too small to determine (carnelian). Decoration: Faience is incised (2 cylindrical, long). Glaze: Mostly worn (faience).

Polish: Moderate (carnelian).

The human(?) figure has hair down to (beyond?) the shoulder, is seated with knees up, with head turned behind him, one hand resting on his knees and one hand on the ground next to him.

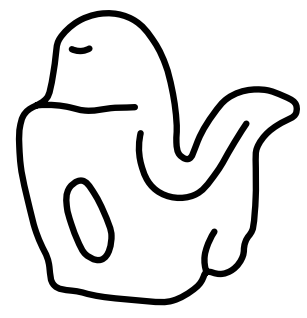


(5) PAHMA 6-14336 Loose beads, some with original string inside

Exactly 36 beads.

Typology: Barrel, short-H15q (amethyst, 1 quartz, 1), H15h (carnelian, 2), PN17f (mud, 1); cylindrical, short-PN22k (faience, 2); cylindrical, long-PN22t (faience, 15); drop-PN22d (faience, 1); ring, cylindrical-PN2c (faience, 5); ball_-PNge (faience, 1), H8f (amethyst, 2), H8d (amethyst, 5). Material: Faience (24); amethyst (8); carnelian (2); quartz (1); mud (1).

Color: Orange (carnelian); green, blue (faience); purple (amethyst); brown (mud); white (quartz). Length: $0.87-17.69 \mathrm{~mm}$.

Diameter: $2.44-7.78 \mathrm{~mm}$. Aperture: $0.36-1.56 \mathrm{~mm}$. Weight: $4.6 \mathrm{~g}$.

Production: OMMA and blue pigment was incorporated in the manufacturing process (faience). No edge.

Aperture: Apex; end.

Perforation: Single plain (2 amethyst); others unknown due to ancient string. Striations: Rotary.

Glaze: Mostly worn ( 7 faience); mostly not worn (17 faience). Polish: Moderate (1 amethyst), dull (amethyst, carnelian, quartz).

For the blue frit used in making some beads being the same as Egyptian blue, see Xia 2014, 40.

(6) РАнма 6-14337 Loose bead

Exactly 1 bead.

Typology: Figural — cowrie shell. Material: Travertine.

Color: White. Length: $16.23 \mathrm{~mm}$. Diameter: $13.53 \mathrm{~mm}$. Aperture: $1.80 \mathrm{~mm}$.

Weight: $1.9 \mathrm{~g}$.

No edge. Aperture: Apex.

Perforation: Not determined because the hole is blocked with string. Striations: Longitudinal.

Decoration: Stone was carved with a groove down the center.

For parallels to the cowrie shell, see Petrie 1914, pl. 14:107 [not numbered on plate]; BM EA 3077; M MA 09.180.1200, 22.1.1279.

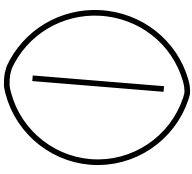

FIGURE 7.8

Cowrie 
(7) РАН ма 6-14338 Loose beads

Exactly 14 beads.

Typology: Disc, barrel—H2k (2); ball—Hgg (12). Material: Carnelian.

Color: Orange, white. Length: 4.83-6.91 mm. Diameter: 5.19-7.34 mm. Aperture: 1.18-2.75 mm. Weight: $4.9 \mathrm{~g}$.

No edge. Aperture: End.

Perforation: Double cone (2 barrel discs); single plain (12 ball beads). Striations: Random.

Polish: Ranges from moderate to high. Original string is still present in 5 ball beads.

(8) PAH MA 6-14339 Loose beads

Exactly 11 beads. Typology: Ball—PN8d. Material: Faience.

Color: Black.

Length: $5 \cdot 72-6.85 \mathrm{~mm}$. Diameter: $5.80-7.53 \mathrm{~mm}$.

Aperture: Could not be measured (too small). Weight: $3.2 \mathrm{~g}$.

Production: SMMD. No edge.

Aperture: End. Glaze: Mostly worn.

(9) PAHMA 6-14340 Loose pendants and a loose bead

Exactly 4 objects (3 pendants and 1 bead).

Typology: Figural-lion (faience, 2); pear-shaped pendants- $\mathrm{H}_{73} \mathrm{r}$ (but larger and with glaze, green feldspar, 1), H37r (amethyst, 1).

Material: Faience (2), amethyst (1), green feldspar (1). Color: Green (feldspar); blue (faience); purple (amethyst). Length of objects: $1.55^{-8.55} \mathrm{~mm}$.

Diameter of objects: $2.71-14.04 \mathrm{~mm}$. Aperture of objects: $0.70-1.20 \mathrm{~mm}$. Weight: $3.1 \mathrm{~g}$.

Production: Molding (faience). No edge.

Aperture: End.

Perforation: Single plain (feldspar, amethyst). Striations: Longitudinal (amethyst); random (feldspar).

Decoration: Imprinted design of the outline of 6 circles arranged in the shape of a cross: 4 circles in a vertical line, and 1 circle on either side of the second vertical circle (feldspar).

Glaze: Mostly worn (feldspar); mostly not worn (faience). Polish: Moderate (amethyst).

For parallels, see the following: Lion: Brunton 1948, pl. 31:25 (15D), 31 (15Z). Feldspar pendant: UC 52186 (shell forehead pendant with 5 rows of circles (not 
just outlines) drilled into it; 3 to 4 circles per row), Uc 52187 (shell forehead pendant with ringed circles incised on the surface, seemingly in the shape of a capital letter I); Petrie 1914, pl. 16:13ok.
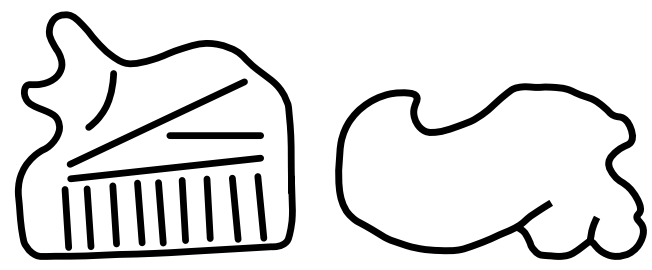

FIGURE 7.9

Lions

(10) PAHMA 6-14341 Loose beads

Approximately 200 beads.

Typology: Disc, cylindrical—PN2j (faience), R52f (ostrich eggshell). Material: Faience (1), ostrich eggshell (approx. 199).

Color: White.

Length: $1.39-1.57 \mathrm{~mm}$. Diameter: $4.10-5.51 \mathrm{~mm}$. Aperture: $1.40-1.81 \mathrm{~mm}$. Weight: $14.8 \mathrm{~g}$.

Production: оммв (faience).

No edge. Aperture: End.

Perforation: Double cone (ostrich eggshell). Glaze: Mostly worn (faience).

\section{(11) PAHMA 6-14343 Strung girdle}

Typology: Disc, barrel—PN2g (only black faience, approx. 720); ring, barrelPN2f (various faience, approx. 1440); disc, cylindrical- $\mathrm{R}_{519}$ (ostrich eggshell, approx. 1080). Material: Faience (approx. 2160), ostrich eggshell (approx. 1080). Color: Light green, light brown, black (faience); white (ostrich eggshell). Length of girdle: $56 \mathrm{~cm}$.

Length of beads: $0.68-2.02 \mathrm{~mm}$. Diameter of beads: $3 \cdot 32-7.01 \mathrm{~mm}$. Production: оммв (faience).

No edge. Aperture: End.

Perforation: Single plain (ostrich eggshell). Glaze: Mostly worn (faience). 


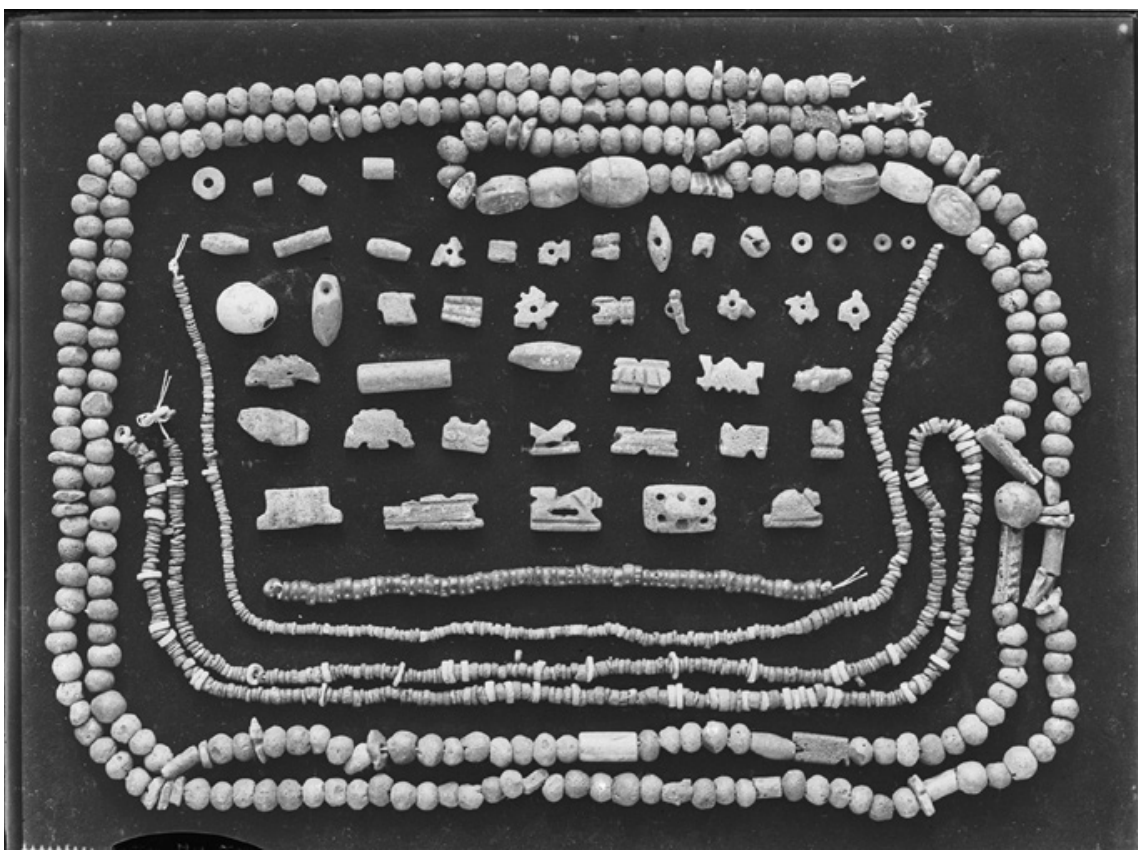

FIGURE 7.10 Beads and pendants from N 2016 and N 2071 (NED-A-8267)

N 2071 Map B4; type rock-cut chamber with sloping entrance; photos C 8074, C 8194, C 8285, C 8567, A 8267, A 8268, B 8250. (Figs. 4.1, 7.10).

This grave had 2 layers of burials, the upper with 4 burials and the lower with 5 burials.

Upper: (a) By pelvis, glaze [barrel] of striped black and white faience. Round neck, string of small brown faience disc beads (PAHMA 6-14372). 1 carnelian [short barrel] 1 [disc], 10o[ed.:?] [short barrel], 2 carnelian [short barrel], and a small scaraboid (PAHMA 6-14372). (b) Round neck string of green faience disc beads (PAHMA 6-14365? PAHMA 6-14368?). Also, a small faience scarab (PAHMA 6-14374). (c) Round neck strings of carnelian short cylindrical beads and tiny faience discs (PAHMA 6-14373? PAHMA 6-14378? РAHMA 6-14379?). Behind head number of faience amulets (PAHMA 6-14375?). By left arm, there had been a small wooden box containing 4 faience scarabs, and a number of faience amulets, and beads [ring] and disc also scattered above body (PAHMA 6-14373?, PAHMA 6-14378?, PAHMA 6-14379?). Number of shells pierced for threading (PAHMA 6-14355, PAHMA 6-14356). Stone pendant with black rings (PAHMA 6-14359). Two faience beads, white with black continuous ring decoration (PAHMA 6-14358). Green faience [ring] and disc beads 
and small amulets (PAHMA 6-14357?, PAHMA 6-14364?, PAHMA 6-14367?). 1 carnelian [cylindrical] and 1 disc (PAHMA 6-14377?). Small shell disc beads (PAHMA 6-14362?). These objects were found with a pottery kohl pot, 2 pieces of an alabaster jar, one of which had been used as a scraper (PAHMA 6-14353, PAH MA 6-14354), pieces of 2 or 3 ivory needles, and 1 mussel shell. In radim above, 2 pieces of a pot, which had been broken and mended anciently.

Lower: By neck of (e), a quantity of beads: blue and black glaze and light stone disc (PAHMA 6-14364?), 1 crumb (PAHMA 6-1436o?, PAHMA 6-14364?), 1 [barrel], 1 glaze scaraboid (PAHMA 6-14363).

[ed.: According to Hearst Museum records, PAHMA 6-14350 (ceramic jar), PAHMA 6-14351, PAHMA 6-14352 (alabaster jar), PAHMA 6-1436o (assorted beads and organic material), PAHMA 6-14361 (bead fragments and rocks), PAHMA 6-14366 (mirror), PAHMA 6-14369 (alabaster jar), PAHMA 6-14370, PAHMA 6-14371 (mud beads and decorations), PAHMA 6-14376 (pendant) are from this tomb.]

(1) PAHMA 6-14355 Loose shells

Exactly 4 shells ( 3 with perforation bored as a pendant and 1 without perforation). Material: Mollusk shell (family: Cardiidae).

Color: Light brown. Length: 4.38-5.68 mm. Diameter: 15.70-19.19 mm. Aperture: $1.50-2.38 \mathrm{~mm}$. Weight $3.0 \mathrm{~g}$.

Perforation: Single plain.

For parallels, see Petrie 1914, pl. 14:111a.

(2) PAHMA 6-14356 Loose beads

Exactly 17 beads.

Typology: Barrel, long (faience, 2; steatite, 4)—PN17h, L12g; ball (faience)— PN8d (small, 7), PN8h (big, 2). Material: Faience (11), steatite (4), mollusk shell (family: Neritidae, 2).

Color: Green (faience); brown (faience, steatite); white (steatite, shell). Length: 4.57-11.6o mm.

Diameter: 4.20-12.70 mm. Aperture: 1.27-4.6o mm. Weight: $6.3 \mathrm{~g}$.

Production: om MA (faience). No edge.

Aperture: End.

Perforation: Single plain (steatite); other (top of shell was cut off to enable shell to be strung as a bead). Striations: Random.

Glaze: Mostly worn (faience, steatite).

For parallels to the shells, see PAH MA 6-1076 from N 3018 (Reisner 1908, 85, 118). 
(3) РАНMA 6-14357 Loose beads

Exactly 23 beads.

Typology: Figural-PDiom (faience spiral, 1); shell? (faience, 1); rosette L66b, L66b', L66d, L66d' (glazed steatite spacers, 11); broken (faience, 10). Material: Faience (10), steatite (13).

Color: Blue (faience); brown (faience, steatite). Length: 1.92-14.66 mm.

Diameter: 4.38-8.45 mm. Aperture: 1.03-1.65 mm. Weight: $2.7 \mathrm{~g}$.

Production: SMMD (faience). No edge.

Aperture: End.

Perforation: Single plain (steatite). Glaze: Mostly worn (faience, steatite).

(4) PAH MA 6-14358 Loose beads (now strung together with wire) Exactly 2 beads.

Typology: Ball_-PD3. Material: Faience.

Color: White with black spiral decoration. Length of beads: 9.56-9.79 mm.

Diameter of beads: 11.73-12.55 mm. Weight: $2.6 \mathrm{~g}$.

Production: OMMA (faience). No edge.

Aperture: Apex. Decoration: Painted. Glaze: Mostly worn.

(5) PAHMA 6-14359 Pendant

Exactly 1 pendant.

Typology: Other (oval drop) -A172.

Material: Quartz, perhaps with ferruginous bands.

Color: Brown, white. Length: $4.61 \mathrm{~cm}$. Diameter: $19.78 \mathrm{~mm}$. Major radius: $17.48 \mathrm{~mm}$.

Width (around thickest point): $8.94 \mathrm{~mm}$. Aperture: $1.56 \mathrm{~mm}$.

No edge. Aperture: End. Perforation: Double cone. Striations: Longitudinal. Polish: Moderate.

(6) PAнма 6-1436o Loose pendants and loose beads

Exactly 6 objects ( 1 broken pendant, 1 intact pendant, 1 broken bead, 3 intact beads).

Typology: Barrel, short-R59 (seed, 1); cylindrical, short-PD5od (faience, 1), L16n (glazed steatite, 1); pendant, lenticular-R46d (mud, 1); broken bead (seed, 1); broken pendant (carnelian, 1).

Material: Faience (1), carnelian (1), steatite (1), mud (1), seed (2). Color: Red (carnelian); brown (faience, glazed steatite, mud, seed). Length of objects: $2.60-12.46 \mathrm{~mm}$. 
Diameter of objects: $4.65^{-7.29} \mathrm{~mm}$. Aperture of objects: $1.03^{-1.5^{2}} \mathrm{~mm}$. Weight: $2.2 \mathrm{~g}$.

Production: OMMA (faience). No edge.

Aperture: End.

Perforation: Single plain (steatite). Striations: Longitudinal (carnelian, steatite). Decoration: Crumb decoration (1 faience). Glaze: Mostly worn (faience, steatite). Polish: Moderate (carnelian).

(7) РАнма 6-14361 Loose bead and rocks

Exactly 4 objects ( 1 bead fragment and 3 rocks).

Typology: Barrel, short (1; no Xia Nai number because broken). Material: Faience (1).

Color: Brown.

Length of bead: $10.41 \mathrm{~mm}$. Diameter of bead: $6.86 \mathrm{~mm}$. Aperture: N/A (broken). Weight: o g (did not register). No edge.

Aperture: End. Glaze: Mostly worn.

(8) PAH MA 6-14362 Loose beads

Approximately 100 beads.

Typology: Disc, barrel-PN6g (faience, approx. 40), R52f (ostrich eggshell, 5); ring, cylindrical-PN6d (faience, approx. 47), H6c (carnelian, 2); spacersL66b, L66d' (steatite, 4), A16 (faience, 1), A207 (carnelian, 1). Material: Faience (approx. 88), carnelian (3), steatite (4), ostrich eggshell (5).

Color: Red (carnelian); green (faience); brown (steatite, faience, unknown); black (faience); white (ostrich eggshell).

Length: $0.75^{-3.25} \mathrm{~mm}$. Diameter: $1.98-6.24 \mathrm{~mm}$. Aperture: $0.64-1.95 \mathrm{~mm}$. Weight: $3.9 \mathrm{~g}$.

Production: om ma (faience). No edge.

Aperture: End.

Perforation: Double cone (carnelian, ostrich eggshell); single plain (steatite, carnelian spacer). Striations: Longitudinal (carnelian); random (steatite). Glaze: Mostly worn (faience). Polish: Moderate, dull (carnelian).

(9) РАнма 6-14364 Loose beads

Exactly 48 beads.

Typology: Barrel, short—PN17f (faience, 19), PD48d (faience with crumb decoration, 8), S14k (steatite, 5); conical, long-Gd7 (glass, 1); cylindrical, longS18s (steatite, 8); ring, barrel—L5b (faience, 1); ball—PN8d (faience, 4); shell (1); broken (faience, 1). 
Material: Faience (33), steatite (13), glass (1), shell (1).

Color: Green (faience); brown (faience, steatite); black (faience); white (steatite). Length: 1.14-14.9 $\mathrm{mm}$.

Diameter: 2.72-6.19 mm. Aperture: 1.00-1.91 mm. Weight: $5.3 \mathrm{~g}$.

Production: oмmA (faience); SMMD (ball bead). No edge.

Aperture: End.

Perforation: Single plain (steatite). Striations: Longitudinal (steatite). Decoration: Crumb decoration (1 faience). Glaze: Mostly worn (faience, steatite).

For a parallel to the crumb bead, see PAнмA 6-14472 (N 2434), UC 51425.

(10) PAнма 6-14365 Loose beads and 1 unperforated shell

Approximately 800 beads.

Typology: Barrel, short-PN17c, PN17f (faience, approx. 5); biconical, shortPN2op (faience, approx. 20); ring, barrel-PN1c (faience, approx. 515), H2a (carnelian, 1); ring, cylindrical-PN2c (faience, approx. 257), R52e (ostrich eggshell, 1); other: unseparated groups of ring, cylindrical-PN62a, PN62a', PN62b (approx. 12 groups).

Material: Faience (approx. 797), carnelian (1), mollusk shell (1 without perforation), ostrich eggshell (1). Color: Orange, green, brown, black, white.

Length of beads: $0.91-6.03 \mathrm{~mm}$. Diameter of beads: 2.57-4.11 mm. Aperture of beads $0.41-0.70 \mathrm{~mm}$.

Weight: $6.7 \mathrm{~g}$.

Production: om mA (faience). Edge and no edge.

Aperture: Apex and end.

Perforation: Double cone (carnelian, ostrich eggshell). Striations: Longitudinal. Glaze: Mostly worn (faience). Polish: Moderate (carnelian).

(11) РАН мА 6-14367 4-strand necklace

Approximately 26o beads.

Typology: Ball-PN8d, PN8f (faience, approx. 232); figural-door/ladder (4, 1 broken), scarabs (4), paddle (1), unknown (ox head?) (1); spacer-PD18d' (but not decorated, 1); cylindrical, short_PN22t (faience, 4); spacer-L66b, L66b', L66d' (steatite, 23); cylindrical, short-L17c (steatite, 1); cylindrical, long_L17e (steatite, 2); barrel, short_L12l (steatite, 2); barrel, long-L11l (steatite, 1); shell—conus shell? perhaps Mitra maculosa shell? (2).

Materials: Faience (approx. 248), steatite (approx. 29), shell (2).

Color: Blue, green (faience); white (faience, steatite, shell); brown (faience, steatite). Length of strand: $38 \mathrm{~cm}$. 
Diameter of strand: $28.2 \mathrm{~cm}$.

Length of beads: $1.48 \mathrm{~mm}-16.74 \mathrm{~mm}$. Diameter of beads: $4.8 \mathrm{o}-10.41 \mathrm{~mm}$. Weight: $51.2 \mathrm{~g}$.

Production: om MA, molding (faience). No edge.

Aperture:Apex, end (faience); end (steatite). Perforation: Single plain(steatite). Striations: Longitudinal (steatite).

Glaze: Mostly worn, mostly not worn (faience); mostly worn (steatite).

For parallels, see the following: Shell: EA 63299; Petrie 1914, pl. 14:109-110. Ox head: Petrie 1914, pl. 5:62; Brunton 1948, pl. 31:41. For a similar looking bull head, MMA 10.130.2050, 10.130.1338. For a design similar to the one reproduced below but on a First Intermediate Period scarab from Matmar, see Brunton 1948, pl. 33:85.

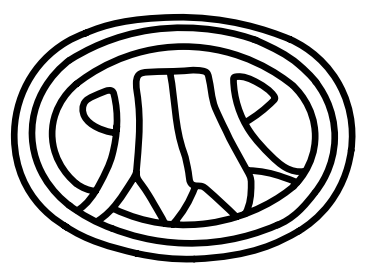

FIGURE 7.11

Scarab

(12) PAHMA 6-14368 Length of necklace (not a loop) Approximately 200 beads.

Typology: Disc, cylindrical-R52f (ostrich eggshell, approx. 42); ring, barrelPN2c (faience, approx. 100); ring, cylindrical-PN6d (faience, approx. 50), R52c (ostrich eggshell, approx. 8); other-PN2d (double ring; faience, 2).

Material: Faience (approx. 150), ostrich eggshell (approx. 5o). Color: Green, brown (faience); white (ostrich eggshell). Length of strand: $5^{2} \mathrm{~cm}$.

Length of beads: $0.71-2.46 \mathrm{~mm}$. Diameter of beads: 2.77-5.76 mm. Weight: $6.5 \mathrm{~g}$. Production: OMMA (faience). No edge.

Aperture: End.

Perforation: Double cone (ostrich eggshell).

Glaze: Mostly worn (faience); mostly not worn (faience).

(13) PAHMA 6-14370 (from N 2071a) Loose beads and conical decoration (without perforation) Approximately 300 objects.

Typology: Barrel, short-PNi7h (approx. 200); cylindrical, short-PN6q (approx. 10); conical decoration-PN19g (approx. 90). The mud is a fine, compact mud. 
Material: Mud. Color: Brown.

Length of objects: $5.32-20.50 \mathrm{~mm}$. Diameter of objects: $2.65^{-5.98} \mathrm{~mm}$.

Aperture of objects: Too small to be measured, if present at all. Weight: $57.0 \mathrm{~g}$. Production: Rolled. No edge.

Aperture: Apex, end, and irregular (some have 1 end side and 1 apex side).

(14) PAHмA 6-14371 (from N 2071a) Loose beads, conical decorations (no perforation), faience ball (no perforation), and chips of shell

Approximately 300 objects.

Typology: Barrel, short-R $57 \mathrm{~d}$ (mud, approx. 170); cylindrical, short- $\mathrm{R}_{58 \mathrm{e}}$ (mud, approx. 30); ring, cylindrical-PN2a, PN2b (faience, 3); ball-PNge (faience, 3 ); other: sharp, conical decoration- $\mathrm{R}_{5} 8 \mathrm{i}$ (mud, approx. 100). The mud is a fine, compact mud.

Material: Faience (ring bead (3), ball bead (3)); mud (approx. 294).

Color: Brown.

Length of objects: $0.77-16.78 \mathrm{~mm}$. Diameter of objects: $2.5^{2-5.81 ~ m m}$.

Aperture of objects: Too small to be measured, if present at all. Weight: $57.7 \mathrm{~g}$. Production: Rolled. No edge.

Aperture: Apex, end, and irregular (some have 1 end side and 1 apex side).

(15) РАн MA 6-14372 Length of string (bracelet?) (not a loop), broken fragments of beads, and a loose scarab

Exactly 200 objects (199 intact beads and 1 scarab).

Typology: Disc, barrel—PN2g (faience, 189); disc, cylindrical-PN6g (faience, 10); figural—scarab (steatite, 1 ).

Materials: Faience (199), steatite (1). Color: Brown.

Length of strand: $21 \mathrm{~cm}$.

Length of beads: $0.79-1.5^{2} \mathrm{~mm}$. Length of scarab: $8.63 \mathrm{~mm}$. Diameter of beads: 2.28-3.68 mm. Diameter of scarab: $3.54 \mathrm{~mm}$. Weight: $1.5 \mathrm{~g}$.

Production: omMA (faience). No edge.

Aperture: End.

Perforation: Single plain (scarab). Striations: Random.

Decoration: Etched (scarab).

Glaze: Mostly worn (faience, glazed steatite).

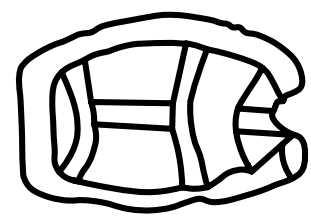

FIGURE 7.12

Scarab 
(16) PAнмA 6-14373 (from N 2071b) Length of necklace (not a loop) with a pendant

Exactly 337 beads and 1 pendant.

Typology: Ring, barrel—PN2g (337); pendant, lenticular (1). Material: Faience. Color: Brown.

Length of strand: $40 \mathrm{~cm}$.

Length of beads: $0.70-1.70 \mathrm{~mm}$. Diameter of beads: $2.87-3.40 \mathrm{~mm}$. Weight: $3.2 \mathrm{~g}$. Production: оммв (faience). No edge.

Aperture: End.

Perforation: Single plain.

Glaze: Mostly worn.

(17) PAHMA 6-14375 (from N 2071c) Loose beads and pendant

Exactly 22 objects ( 21 beads and 1 pendant).

Typology: Barrel, short (blobbed) - $\mathrm{PN}_{17} \mathrm{~h}$ (faience, 1); lenticular- $\mathrm{PN}_{32} \mathrm{f}$ (faience, 1); spacers (steatite)—L66b (1), Lbb6' (2), L66d (1), L66d' (2); figural (faience) — ram/ichneumon (1), winged scarab (2), door/ladder (1), lion (1), double lion head (1), double lion (1), with horizontal lines (4), broken (2); sixholed pendant (1). Material: Faience (16), steatite (6).

Color:Blue (faience); brown(faience, steatite). Length of objects:1.97-18.95 mm. Diameter of objects: 4.07-10.14 mm. Aperture of objects: $0.94-1.57 \mathrm{~mm}$. Weight: $5 \cdot 3 \mathrm{~g}$.

Production: омма (faience: barrel, short), омм в (faience: lenticular), molding (faience: figurals).

No edge. Aperture: End. Perforation: Single plain.

Striations: Longitudinal (1 spacer), indeterminate (5 spacers).

Glaze: Mostly worn (faience, steatite).

The blobs on the faience bead $\mathrm{PN}_{17} \mathrm{~h}$ are regular and irregular. Glaze clearly overlays the blobs, so the blobs appear to have been formed during the firing process. For parallels, see the following: Ram/Ichneumon: Reisner 1907, pl. 21:12337 (listed as a ram); Brunton 1948, pl. 31:45-46 (listed as an ichneumon). Winged scarab: Petrie 1914, pl. 11:93d-e; UC43123. Door/ladder: MMA 22.1.1944j; Reisner 1923, 123, pl. 43,2. Lion: Brunton 1928, pl. 95:15D3, 15D6. Double lion head: Brunton 1928, pl. 95:17D6; Petrie 1914, pl. 10:91c (although it is gold); Reisner 1907, pl. 18:12225 (frit). Double lion: Brunton 1928, pl. 95:17D6. Six-holed pendant: Petrie 1914, pl. 33:188z; Brunton 1948, pl. 32:82-84, 105. 
(18) PAHMA 6-14376 (from N 2071c) Loose pendant Exactly 1 pendant.

Typology: Pendant, lenticular- $-\mathrm{H} 74 \mathrm{j}$. Material: Agate.

Color: Red, orange, white. Length: $3.35 \mathrm{~mm}$. Diameter: $6.12 \mathrm{~mm}$.

Top to bottom: $15.10 \mathrm{~mm}$. Aperture: $1.03 \mathrm{~mm}$. Weight: $0.7 \mathrm{~g}$.

No edge. Aperture: End. Perforation: Double cone. Striations: Random. Polish: Moderate.

(19) PAHMA 6-14377 (from N 2071c) Loose beads and one shell without a perforation

Exactly 78 objects.

Typology: Barrel, long-PNi7l (faience, 8); cylindrical, short—Li6j (steatite, 3); cylindrical, long- $\mathrm{L} 16 \mathrm{q}$ (steatite, 2); disc, cylindrical- $\mathrm{R}_{52 \mathrm{f}}$ (ostrich eggshell, 25); ring, barrel—PN2g (faience, 20), H2f (carnelian, 7); ball—PN8d (faience, 6); other: broken faience (1); double elliptical with teardrop-shaped perforation-PN14C (faience, 1); spacer bead-PD17e (faience, 1); 2 shells, family: Neritidae; 1 shell, family: Conidae; 1 shell, type undetermined (top had been cut off in order to string it).

Material: Faience (37), carnelian (7), steatite (5), mollusk shell (4), ostrich eggshell (25). Color: Orange, green, brown, white.

Length of objects: 1.13-19.32 mm. Diameter of objects: 2.00-10.40 mm. Aperture of objects: $0.8 \mathrm{o}-3.32 \mathrm{~mm}$. Weight: $7 \cdot 3 \mathrm{~g}$.

Production: OMMA (faience). No edge.

Aperture: End.

Perforation: Double cone (carnelian, ostrich eggshell); single plain (glazed steatite, mollusk shells). Striations are random.

Glaze: Mostly worn (faience, steatite). Polish: Moderate (carnelian).

(20) PAHмA 6-14378 (from N 2071c) Length of bracelet (not a loop) Exactly 69 beads.

Typology: Disc, barrel—H2e (37); disc, biconical- $\mathrm{H}_{5} \mathrm{~d}$ (6); disc, cylindricalH6e (24); ring, cylindrical- $\mathrm{H6c}(2)$.

Material: Carnelian. Color: Red, orange.

Length of strand: $12.5 \mathrm{~cm}$. Length of beads: $0.92-2.3^{1} \mathrm{~mm}$. Diameter of beads: 2.58-4.59 mm. Weight: $2.7 \mathrm{~g}$.

Edge (disc, biconical); no edge (the remainder of the beads). Aperture: End.

Perforation: Double cone. Striations: Rotary.

Polish: High. 
(21) PAH MA 6-14379 (from N 2071c) Length of necklace (not a loop) Exactly 283 beads.

Typology: Disc, cylindrical—PN6g (faience, approx. 50); ring, barrel-PN2c (faience, approx. 231); ring, biconical— $\mathrm{H}_{5} \mathrm{e}$ (carnelian, 1); figural—ring bead with knob (faience, 1 ).

Material: Faience (282), carnelian (1).

Color: Red (carnelian); blue, brown, black (faience). Length of strand: $29 \mathrm{~cm}$.

Length of beads: $0.52-1.96 \mathrm{~mm}$. Diameter of beads: $2.14-3.32 \mathrm{~mm}$. Weight: $1.8 \mathrm{~g}$. Production: омma (faience). No edge.

Aperture: End.

Perforation: Double cone (carnelian). Glaze: Mostly worn (faience).

Polish: Moderate (carnelian).

N 2072 Map A5; photo C 8235

Round neck, several strings of glaze beads sizes apparently strung separately. In front, probably on shell necklace, lion plaque in limestone and 1 carnelian [long barrel] bead (PAHMA 6-14381; for the lion plaque, see also Reisner 1932, fig. 50:19). Also, string of black glaze beads. In front, apparently on black string, carnelian [short barrel] and [long barrel], limestone [cylindrical], and quartzite [long barrel] (PAHMA 6-14380, PAHMA 6-14381). With these finds were several broken As and 1 jar.

(1) PAHMA 6-1438o Loose beads

Approximately 175 beads.

Typology: Conical, short-PN21e (faience, 1); disc, cylindrical-PN6d, PN6g (faience, approx. 139), H6e (carnelian, 9), R52e (ostrich eggshell, approx. 25); figural-lion (faience, 1).

Material: Faience (approx. 141), carnelian (9), ostrich eggshell (approx. 25).

Color: Red, orange (carnelian); green, brown, black (faience); white (faience, ostrich eggshell). Length: $0.48-7.67 \mathrm{~mm}$.

Diameter: 2.18-6.15 mm. Aperture: 1.10-2.03 mm. Weight: 4.1 g.

Production: омMA, molding (faience). No edge.

Aperture: End.

Perforation: Double cone (carnelian); single plain (ostrich eggshell). Striations: Random.

Glaze: Mostly worn (faience). Polish: Moderate, dull (carnelian).

For a parallel to the lion, see Brunton 1928, pl. 95:15D6. 
(2) PAH MA 6-14381 Length of necklace (not a loop)

Approximately 370 beads.

Typology: Barrel, short-H15n (carnelian, 1); conical, short-PNigd (faience, 4); cylindrical, short-S18i (steatite, 1); disc, barrel- $\mathrm{H} 2 \mathrm{k}$ (quartz, 1); disc, biconical $-\mathrm{H}_{5 j}$, $\mathrm{H}_{5} \mathrm{~m}$ (carnelian, 2); disc, cylindrical- $\mathrm{R}_{52 \mathrm{f}}$ (ostrich eggshell, approx. 270); ring, cylindrical- $\mathrm{R}_{52 \mathrm{c}}$ (ostrich eggshell, approx. 89); figurallion; broken (faience, 2). Material: Faience (6), carnelian (3), quartz (1), steatite (1), ostrich eggshell (approx. 359).

Color: Red (carnelian); brown (faience); white (quartz, steatite, ostrich eggshell). Length of strand: $47.5 \mathrm{~cm}$.

Length of beads: $0.67-6.88 \mathrm{~mm}$. Diameter of beads: $3.86-9.28 \mathrm{~mm}$. Weight: $17.3 \mathrm{~g}$. Production: omma (faience), molding (lion) Edge (carnelian); no edge (the remainder). Aperture: End.

Perforation: Double cone (quartz, carnelian); single plain (steatite, ostrich eggshell). Striations: Longitudinal (steatite); rotary (carnelian); random (quartz). Glaze: Mostly worn (faience, steatite). Polish: Dull (carnelian), rough (quartz).

For parallels to the lion, see Brooklyn Museum 16.366; Petrie 1914, pl. 38:219; Reisner 1923, 128, pl. 44,2,20-21.

N 2075 Map A4; type vi d; photo C 8o74, C 8194, B 8248

By neck, quantity of carnelian beads scattered. Barrel carnelian strung with black glaze disc between; in one place, a lazuli disc in place of glaze (PAHMA 6-14389a, PAHмA 6-14389b, PAHMA 6-1439o). Small carnelian [ring bead] strung together (PAHMA 6-14385). Layer of same shape either together or with black glaze between. Also, a string of tiny shell (РAHMA 6-14385). In large chamber, few shell disc beads.

These items were found with, in main chamber, a few pots, mostly broken. Piece of a drab [bowl]? 2 alabaster C 8194 1/5 [squat jar] and $2 / 5$ [jar]. [ed.: According to Hearst Museum records, PAHMA 6-14382 (glass bead/plug), PAHMA 6-14383 (scarab), PAHMA 6-14384 (assorted beads, including a striped faience bead similar to the drawing in 2071), PAHMA 6-14384.1 (metal beads), PAHMA 6-14386 (jar fragment), PAHMA 6-14387 (metal wire), PAHMA 6-14388 (needle), PAHMA 6-14391, PAHMA 6-14392 (alabaster vessels), PAHMA 6-14393, PAHMA 6-14394, PAHMA 6-14395 (mirrors), PAHMA 6-14396 (ceramic jar) are from this tomb.]

(1) Pahma 6-14382 Loose bead

Exactly 1 bead.

Typology: "Ear stud," but pierced from flat end to tip so as to be strung.

Material: Glass. 
Color: Yellow, blue, white. Length: $36 \mathrm{~mm}$.

Diameter at center: $7.15 \mathrm{~mm}$. Diameter at flat end: $16.11 \mathrm{~mm}$. Diameter at tip: $8.36 \mathrm{~mm}$. Weight: $4.5 \mathrm{~g}$.

Production: Modeling (glass). No edge.

Aperture: End. Perforation: Single plain.

Decoration: By simple elements without a separate matrix; left spiral white on blue with yellow on base and tip.

For parallels, see вм ЕА 68532, 68535; MмA 21.9.321; SI F19o9.446; Brooklyn Museum 48.66.30, 48.66.31, 37.1438Ea-b; Andrews 1997, p. 117, fig. 96g; Petrie 1914, p. 12-13, pl. 4:20; Reisner 1907, p. 125:12141, 12143, pl. 10:12141.

(2) PAHMA 6-14384, PAHMA 6-14384.1 Loose beads and a seed Exactly 15 objects.

Typology: Barrel, short—H15m (carnelian, 1; glazed steatite, 2), M7d (gold, 1); barrel, long-PDie (faience, 1); cylindrical, long-PD62b (faience, 1), PN22t (faience, 1); disc, cylindrical—H6e (carnelian, 1); ball—PN8d (faience, 1), PN8h (faience, 2); figural-(faience) fly(?) (1); other: lenticular-PN33d (1); N/A (broken steatite, 1 ; seed, 1 ).

Material: Faience (8), carnelian (2), steatite (3), gold (1), seed (1).

Color: Orange (carnelian), green (faience), brown (faience, steatite), black (faience stripe), rose gold (gold). Length of beads: 1.53-18.75 mm.

Diameter of beads: $4.20-10.10 \mathrm{~mm}$. Aperture of beads: $1.10-1.90 \mathrm{~mm}$. Weight: $3.7 \mathrm{~g}$.

Production: омма (faience), molding (ribbed cylindrical, long and figural (fly?)); joined-halves (metal).

No edge. Aperture: End.

Perforation: Double cone (carnelian barrel); single plain (carnelian disc, all steatite, gold). Striations: Random.

Decoration: Painted (1 with black spiral) (faience). Glaze: Mostly worn (faience, glazed steatite). Polish: Moderate (carnelian).

For parallels to the fly, see Petrie 1914, pl. 2:19j; Reisner 1923, 131. Object PAHMA 6-14384.1 (metal bead) is strung on modern string and stored with PAHMA 6-11575, fragments of a gold ring from Tomb 3576 (Cemetery N 350o).

(3) PAHмA 6-14385 Some loose beads and a length of bracelet (not a loop) Approximately 150 beads.

Typology: Ring, barrel—He (carnelian, 6), PNic (faience, approx. 10o); cylindrical, long-S18s (steatite, 1), PN22t (faience, 1); disc, barrel—PN2j (faience, approx. 50). 
Material: Faience (approx. 143), steatite (1), carnelian (6).

Color: Orange (carnelian); brown (faience); black (faience); white (steatite). Length of beads: $0.88-15.74 \mathrm{~mm}$.

Diameter of beads: $2.27-4.88 \mathrm{~mm}$. Aperture of beads: $0.53^{-1.95} \mathrm{~mm}$. Weight: $3.4 \mathrm{~g}$.

Production: оммв (faience). No edge.

Aperture: End.

Perforation: Double cone (carnelian); single plain (steatite). Striations: Longitudinal (steatite).

Polish: Moderate (carnelian). Glaze: Mostly worn (faience).

(4) PAHMA 6-14389a Necklace (not as originally strung; see PAHMA 6$14389 \mathrm{~b})$ Exactly 22 beads.

Typology: Barrel, short-H15p, H15q (carnelian and agate, 13); barrel, long H16e (carnelian, 1); biconical, short—High (carnelian and agate, 6); biconical, long-H19n (agate, 1); drop-H2op (agate, 1).

Material: Carnelian (17), agate (5).

Color: Red, orange (carnelian, agate); white (agate). Length of strand: $16.4 \mathrm{~cm}$.

Diameter of strand: $11.9 \mathrm{~cm}$. Length of beads: $9.15^{-20.16 ~ m m}$. Diameter of beads: $6.75^{-12.48} \mathrm{~mm}$. Weight: $34.4 \mathrm{~g}$.

Edge and no edge (random; does not correlate with material or shape). Aperture: End.

Perforation: Double cone (carnelian, agate); single cone (drop agate); single plain (carnelian). Striations: Rotary (carnelian, agate).

Polish: High (agate); moderate, dull, rough (carnelian).

For parallels, see PAHмA 6-1050 from N 3061 (Reisner 1908, 85-86); PAHMA 6-10465 from $\mathrm{N}_{5} \mathrm{O} 3$.

(5) PAHMA 6-1438gb Necklace (not as originally strung; these beads were found with PAHMA 6-14389a and with them, may have comprised 1 necklace) Exactly 273 beads.

Typology: Cylindrical, long (carnelian, 1)—H21q; disc, biconical (carnelian, approx. 118)- $\mathrm{H}_{2}$, $\mathrm{H}_{5} \mathrm{e}$ (small), $\mathrm{H}_{2} \mathrm{i}, \mathrm{H}_{5} \mathrm{i}$ (medium), $\mathrm{H}_{2} \mathrm{k}, \mathrm{H}_{5}$ m (large); ring, barrel (carnelian, approx. 155; agate, 3; faience, 4; Egyptian blue, 1)-PN2g.

Material: Faience (4), carnelian (264), agate (3), Egyptian blue (1).

Color: Red, orange (carnelian); blue (Egyptian blue); black (faience); white (agate). Length of strand: $73.0 \mathrm{~cm}$.

Diameter of strand: $26.0 \mathrm{~cm}$. Length of beads: $1.68-4.38 \mathrm{~mm}$. Diameter of beads: $3.98-7.09 \mathrm{~mm}$. Weight: $29.2 \mathrm{~g}$. 
Production: оммв (faience).

Edge (carnelian disc and barrel beads); no edge (carnelian cylinder, faience, agate, Egyptian blue). Aperture: End.

Perforation: Double cone (carnelian, agate); single plain (carnelian cylinder). Striations: Rotary (agate); random (carnelian).

Glaze: Mostly worn (faience).

Polish: High, moderate (carnelian, agate).

(6) PAHMA 6-1439o Loose beads and broken faience ring and disc beads Exactly 12 intact beads and many broken faience beads.

Typology: Barrel, short—H2r (carnelian, 5), Hgg (carnelian, 2); barrel, longH15p (carnelian, 3; quartz, 1)—H15p; disc, cylindrical—H2i (carnelian, 1); broken ring and disc beads (faience).

Material: Faience (no count because broken), quartz (1), carnelian (11). Color: Red, orange (carnelian); black (faience); white (quartz).

Length of beads: $2.58-8.43 \mathrm{~mm}$. Diameter of beads: 5.74-10.50 mm. Aperture of

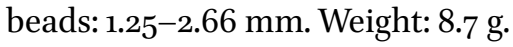

No edge. Aperture: end.

Perforation: Double cone (carnelian, quartz).

Striations: Rotary (carnelian), random (carnelian; quartz). Glaze: Mostly worn (faience).

Polish: High (1 carnelian); moderate (10 carnelian, 1 quartz).

\section{N 209o Map C5; photo A 8266, B 7146}

Bead girdle 8 strings. Behind back, order from above was white shell (1), white and black (2), white shell (3), white shell (5), white and black (4), white and black (7), green (8), white (6). 7 and 8 did not go over left ilium. 7 came round underneath into position next to 4 . 8 went over 6 and under 7 and 4 and came up between 7 and 6 . Under right ilium, order from above was 1234 567 8. All strings went over right ilium. Strings were not fastened together in any way as strings crossed especially at sides where they kept no sort of order. By right arm, few strings[?] green glaze and shell beads and 1 carnelian possibly bracelet. Round neck, string of tiny glaze disc beads. Also, a few of the beads by neck, but these may have come from girdle as beads were much scattered (PAHMA 6-14397, PAHMA 6-14398, PAHMA 6-14399, PAHMA 6-1440O, PAHMA6-14401,PAHMA 6-14402,PAHM 6-14403,PAH MA6-14404,PAHMA6-14405, PAHMA 6-14407). [ed.: According to Hearst Museum records, PAHMA 6-14406 (carnelian djed-pillar pendant) and PAHMA 6-14408 (pot stand) are from this tomb.] 
(1) PAHMA 6-14397 Necklace Approximately 270 beads. Typology: Disc, cylindrical- $\mathrm{R}_{52 \mathrm{f}}$. Material: Ostrich eggshell.

Color: White.

Length of strand: $36 \mathrm{~cm}$. Diameter of strand: $25.5 \mathrm{~cm}$. Length of beads: 1.33-1.92 mm. Diameter of beads: 5.04-7.01 mm. Weight: $37.3 \mathrm{~g}$.

No edge. Aperture: End. Perforation: Double cone.

(2) PAH MA 6-14398 Necklace

Exactly 494 beads.

Typology: Disc, barrel—PN2g (faience); disc, cylindrical— $\mathrm{R}_{52 \mathrm{~m}}$ (ostrich eggshell). Material: Faience (429), ostrich eggshell (65).

Color: Black (faience); white (ostrich eggshell). Length of strand: $68 \mathrm{~cm}$.

Length of beads:0.90-2.05 mm. Diameter of beads: $4.04-5.65 \mathrm{~mm}$. Weight:18.5 g. Production: OMMA (faience). No edge.

Aperture: End. Glaze: Mostly worn.

(3) PAHMA 6-14399 Necklace Approximately 342 beads.

Typology: Disc, cylindrical—R ${ }_{52 f}$. Material: Ostrich eggshell.

Color: White.

Length of strand: $31.4 \mathrm{~cm}$. Diameter of strand: $21.4 \mathrm{~cm}$. Length of beads: 1.58-2.19 mm. Diameter of beads: $6.46-8.27 \mathrm{~mm}$.

Weight: Approximately $22 \mathrm{~g}$ (strung too tightly to measure completely on scale).

No edge. Aperture: End. Perforation: Single plain.

(4) PAHMA 6-1440O Length of necklace (not a loop) Exactly 491 beads.

Typology: Disc, barrel (faience, approx. 30)—PN6k; disc, cylindrical (ostrich eggshell, 151)—R52f; ring, barrel (faience, approx. 310)—PN2j.

Material: Faience (340), ostrich eggshell (151). Color: Black (faience), white (eggshell).

Length of strand: $71 \mathrm{~cm}$.

Length of beads: $0.97-1.87 \mathrm{~mm}$. Diameter of beads: 3.34-5.70 mm. Weight:17.6 $\mathrm{g}$. Production: омma (faience). No edge.

Aperture: End.

Perforation: Single plain (eggshell). Glaze: Mostly worn (faience).

(5) PAH MA 6-14401 Necklace Approximately 308 beads. Typology: Disc, cylindrical—R ${ }_{52 f}$. Material: Ostrich eggshell. Color: White. 
Length of strand: $29.1 \mathrm{~cm}$. Diameter of strand: $18.7 \mathrm{~cm}$. Length of beads: o.49-2.25 mm. Diameter of beads: 4.58-6.97 mm.

Weight: approx. $15.9 \mathrm{~g}$ (strand too large for accurate reading on scale). No edge. Aperture: End. Perforation: Single plain.

(6) PAнма 6-14402 Length of necklace (not a loop) Exactly 304 beads. Typology: Disc, cylindrical—R52f. Material: Ostrich eggshell.

Color: White.

Length of strand: $59.0 \mathrm{~cm}$. Length of beads: $0.78-1.94 \mathrm{~mm}$. Diameter of beads: 6.22-7.56 mm. Weight: $33.4 \mathrm{~g}$.

No edge. Aperture: End. Perforation: Single plain.

(7) PAH MA 6-14403 Length of necklace (not a loop) Exactly 563 beads. Typology: Disc, barrel—PN2f (faience, approx. 6o); disc, cylindrical—PN6k (faience, 121), R52f (ostrich eggshell, 127); ring, barrel—PN2b (faience, approx. 255). Material: Faience (436); ostrich eggshell (127).

Color: Black (faience); white (ostrich eggshell). Length of strand: $78 \mathrm{~cm}$.

Length of beads: $0.82-1.81 \mathrm{~mm}$. Diameter of beads: $4.61-5.60 \mathrm{~mm}$. Weight: $22.1 \mathrm{~g}$. Production: омma (faience). No edge.

Aperture: End.

Perforation: Single plain (ostrich eggshell). Glaze: Mostly worn (faience).

(8) PAH MA 6-14404 Length of necklace (not a loop), with some loose beads Exactly 366 beads ( 360 strung and 6 loose).

Typology: Disc, barrel—PN2k (approx. 272); disc, cylindrical—PN6h (3); ring, barrel—PN2g (approx. 91). Material: Faience.

Color: Green, brown. Length of strand: $5^{8 \mathrm{~cm}}$.

Length of beads: $1.15^{-2.45} \mathrm{~mm}$. Diameter of beads: $4.01-4.69 \mathrm{~mm}$. Weight: $10.9 \mathrm{~g}$. Production: om Ma (faience). No edge.

Aperture: End. Glaze: Mostly worn.

(9) PAнma 6-14405 Some loose beads and length of bracelet (not a loop) Exactly 200 beads (180 strung and 20 loose).

Typology: Disc, cylindrical—R52e (ostrich eggshell, 124); ring, barrel—PN2g (faience, 74); ring, biconical- $\mathrm{H}_{5 j}$ (carnelian, 1).

Material: Faience (74); carnelian (1); ostrich eggshell (124).

Color: Orange (carnelian); green (faience); brown (faience); white (ostrich eggshell). Length of strand: $21 \mathrm{~cm}$.

Length of beads: $0.89-2.03 \mathrm{~mm}$. Diameter of beads: $2.64-4.02 \mathrm{~mm}$. Aperture of beads: $0.70-1.67 \mathrm{~mm}$. Weight: $3.4 \mathrm{~g}$. 
Production: OmMA (faience).

Edge (carnelian); no edge (all the rest). Aperture: End.

Perforation: Double cone (carnelian); single plain (ostrich eggshell). Striations: Random.

Glaze: Mostly worn (faience); mostly not worn (a few green faience). Polish: Dull (carnelian).

(10) PAHMA 6-14406 Loose pendant

Exactly 1 pendant.

Typology: Figural—djed pillar. Material: Carnelian.

Color: Orange. Length: $10.75 \mathrm{~mm}$. Width: $21.63 \mathrm{~mm}$.

Length of aperture (outside): $2.76 \mathrm{~mm}$. Width of aperture (outside): $2.92 \mathrm{~mm}$. Aperture (inside): $1.82 \mathrm{~mm}$.

Weight: 2.5 g. No edge. Aperture: End.

Perforation: Double cone. Striations: Longitudinal. Decoration: Carved. Polish: Moderate.

For parallels, see Petrie 1914, pl. 3:35a; Reisner 1923, 123, pl. 43,2.

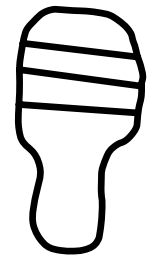

FIGURE 7.13

Djed pillar

(11) PAHMA 6-14407 Length of necklace (not a loop) Approximately 734 beads.

Typology: Ring, cylindrical—PN2c. Material: Faience.

Color: Blue, brown. Length of strand: $36.7 \mathrm{~cm}$. Length of beads: 1.07-1.31 mm.

Diameter of beads: $2.91-3.14 \mathrm{~mm}$. Weight: 3.0 g.

Production: оммв (faience). No edge.

Aperture: End. Glaze: Mostly worn.

N 2092 Map: $\mathrm{C}_{5}$

Round neck, double string of small blue faience disc beads (PAHMA 6-14410).

(1) PAHMA 6-14410 Length of necklace (not a loop) Approximately 1,076 beads.

Typology: Ring, barrel-PNib, PN2b, PN2c (faience, approx. 1,074); ring, cylindrical—PN62a (2 bright blue faience that are stuck together). 
Material: Faience.

Color: Green, blue, brown, white. Length of strand: $83 \mathrm{~cm}$.

Length of beads: $0.5^{2-1.27} \mathrm{~mm}$. Diameter of beads: $2.67-3.62 \mathrm{~mm}$. Weight: $8.8 \mathrm{~g}$. Production: OMMA (faience). No edge.

Aperture: End.

Glaze: Mostly not worn ( 2 bright blue faience beads that are stuck together); mostly worn (all of the other beads).

N 2093 Map C5; photo B 7129

In large chamber, shell which had been painted (PAHMA 6-14413). This object was found with a broken stela, several As, M, [jars] (PAHMA 6-14411, PAHMA 6-14416), small clay kohl pot (PAHMA 6-14418?), broken stem of an alabaster vase (PAHMA 6-14417), granite paint grinder and pebble (PAHMA 6-14414), and small alabaster [jar] (PAHMA 6-14412). In chamber b, 2 A pots. [ed.: According to Hearst Museum records, PAH MA 6-14415 (flint knife) is from this tomb.]

(1) РАНMA 6-14413 Loose shell without perforation

Exactly 1 object.

Material: Mollusk shell (family: Cardiidae). Color: Light brown.

Length: $13.80 \mathrm{~mm}$. Diameter: $33.33 \mathrm{~mm}$. Weight 13.5 g. Perforation: None.

Shell length and diameter measured as if the shell had a perforation in the same way as did 3 of the shells of object number PAH MA 6-14355. For a parallel, see Petrie 1914, pl. 14:111a.

$\mathrm{N}_{2105}$ Map D4

This object not mentioned in the excavation record of $\mathrm{N} 2105$. In that tomb, a mirror (PAHMA 6-14436) was found. [ed.: According to Hearst Museum records, PAHMA 6-1411 (broken ceramic dish) is also from this tomb.]

(1) PAнма 6-14437 Loose beads

Exactly 3 beads.

Typology: Barrel, short-H15m (carnelian, 2); cylindrical, long-PN22t (faience, 1). Material: Faience (1), carnelian (2).

Color: Orange (carnelian); brown (faience). Length: 5.50-12.43 mm.

Diameter: 3.84-6.11 mm. Aperture: 1.72-2.52 mm. Weight: $0.7 \mathrm{~g}$.

Production: OMMA (faience). No edge.

Aperture: End.

Perforation: Double cone (carnelian). Striations: Rotary (carnelian).

Glaze: Mostly worn (faience). Polish: Moderate (carnelian). 


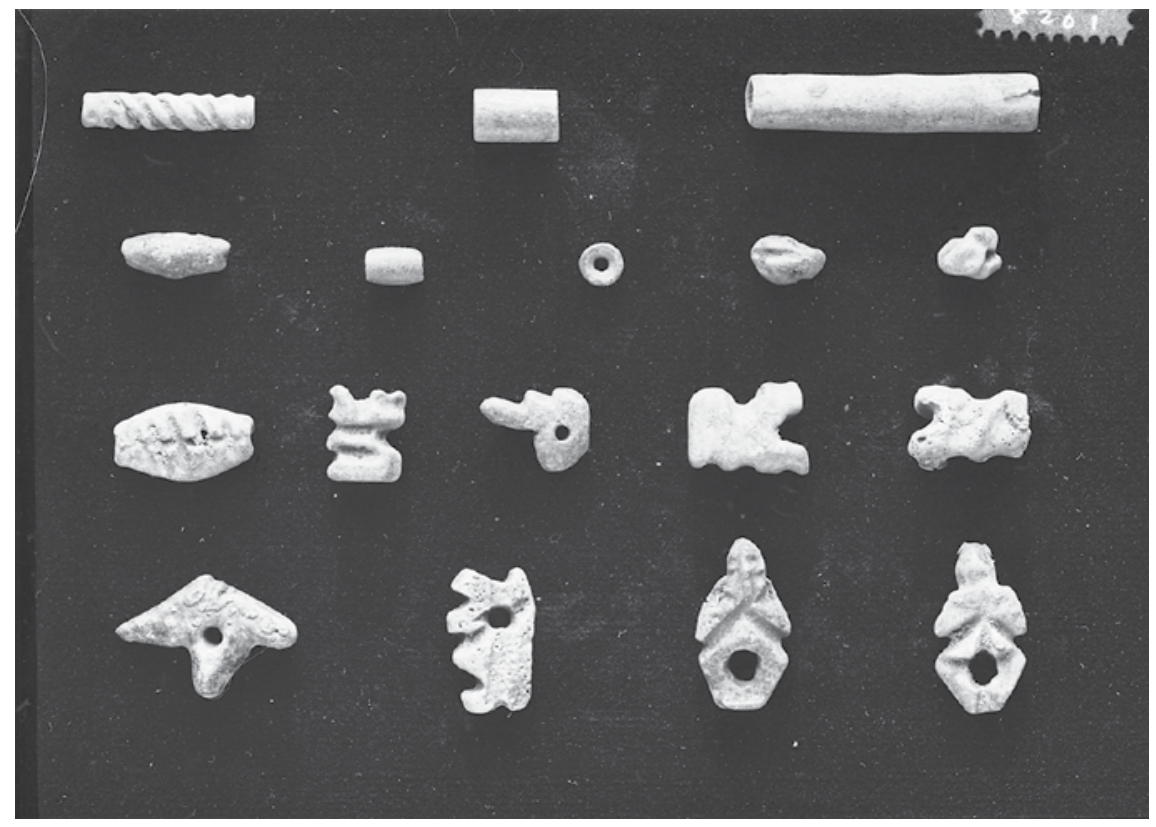

FIGURE 7.14 Beads and pendants from N 2212 (NED-C-82O1)

N 2212 Map D5 (red); photo C 8201 (Fig. 7.14)

By upper part [ed.: Of coffin? Because upper part of body gone.], remains of necklace of, in faience, 1. "was" head amulet, 2. Bes eating snakes, 3. sphinx amulets, frog, stone [cylindrical], carnelian [cylindrical] and disc, 3 shells. In filling, pieces of or saucers of $M$ type. [ed.: Note that Hearst Museum records erroneously attributed these objects to $\mathrm{N}$ 2112.]

\section{(1) PAHMA 6-14440 Loose pendants and beads}

Exactly 15 beads and 6 pendants.

Typology: Barrel, short—H15m (carnelian, 1); cylindrical, short—L17c (steatite, 1); cylindrical, long-PN22v, PD1op (faience, 2); figural (faience) — 2 Bes (?), 2 lions, 1 paddle, 1 frog, 1 was-scepter head, 1 Tawaret (?), 1 unknown (faience); (steatite)—1 lion head; other (shells, 3; broken faience pieces, 4).

Material: Faience (15), steatite (2), carnelian (1), shell (3).

Color: Red (carnelian); blue (faience); brown (steatite); white (shell). Length of objects: $4.93^{-29.46 ~} \mathrm{~mm}$.

Diameter of objects: $3.81-14.00 \mathrm{~mm}$. Aperture of objects: $1.21-4.81 \mathrm{~mm}$. Weight: $8.5 \mathrm{~g}$.

Production: OMMA (faience), molding (faience). No edge. 
Aperture: End.

Perforation: Single plain (steatite, carnelian). Striations: Longitudinal (steatite); random (carnelian). Decoration: Incised (1 faience cylinder); carved (steatite). Glaze: Mostly not worn (faience, steatite).

Polish: Dull (carnelian).

For parallels, see the following: Bes: MMA 11.215.178; Petrie 1914, pl. 33:188; Brunton 1948, pl. 31:9. Lion head: UC 18117; B M EA 57774i (Andrews 1997, fig. 5e); UC 38551; Petrie 1914, pl. 2:22f; Brunton 1928, pl. 95:16N9, 1948, pl. 31:33-34; PAHMA 6-10067 from N 570. Lion: Brooklyn Museum 16.366; Brunton 1948, pl. 31:24-25; Petrie 1914, pl. 38:219; Reisner 1923, 128, pl. 44,2,20-21.
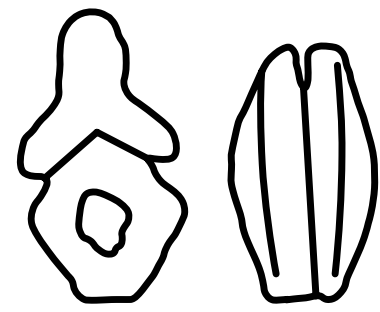

N 2235 Map E4; photos C 8193, C 996, B 7114, B 8251 (Fig. 7.1)

In large chamber, a number of small clay discs (PAH MA 6-14456) and a quantity of glaze disc beads (PAHMA 6-14457). [ed.: According to Hearst Museum records, PAHMA 6-14462 (assorted amulets and beads) are from this tomb.] These objects were found with a quantity of pottery, about 4 As [jars] (PAHMA 6-14451 a-b, РАНMA 6-14452, РАНMA 6-14453, РАНMA 6-14454, РАНMA 6-14455, PAHMA 6-14458), M with white rings [jars].

(1) PAHMA 6-14456 Loose sequins

Exactly 325 objects.

Typology: PN8d, except without perforation. The mud is a fine, compact mud. Material: Mud; a few rocks, most of which are small. Color: Brown; white (a few rocks).

Weight: 64.9 g.

Production: Sequins are flat on the bottom and rounder on top, seemingly not formed by pressing down on the top with a finger.

This object is accompanied by a small bag of fine mud crumbles. 
(2) PAHMA 6-14457 Length of necklace (not a loop) Approximately 1,140 beads.

Typology: Ring, cylindrical and barrel—PN2c.

Material: Faience. Color: Black.

Length of strand: $60 \mathrm{~cm}$. Length of beads: $0.67-3.39 \mathrm{~mm}$. Diameter of beads: $2.66-4.36 \mathrm{~mm}$.

Weight: $4.7 \mathrm{~g}$.

Production: оммв (faience). No edge.

Aperture: End. Glaze: Mostly worn.

(3) PAHMA 6-14462 Length of bracelet (not a loop) Exactly 9 beads.

Typology: Spacer-PD18d' (although undecorated; faience, 1); figural (faience) - lion heads (4), tj.t amulet (1), lion (1); figural (coral?) —lion (1).

Material: Faience (7), coral(?) (1), shell (1).

Color: Green, brown (faience); white (coral, shell). Length of beads: 3.06-12.57 mm.

Diameter of beads: $7.26-13.24 \mathrm{~mm}$. Weight: $3.1 \mathrm{~g}$.

Production: Molding (faience). No edge.

Aperture: End.

Perforation: Single plain (coral). Striations: Random.

Glaze: Mostly worn (faience).

For parallels, see the following: tj.t amulet, Petrie 1914, pl. 7:88. Lion: Brooklyn Museum 16.366; Petrie 1914, pl. 38:219; Reisner 1923, 128, pl. 44,2,20-21. Lion head: UC 18117; BM EA 57774i (Andrews 1997, fig. 5e); UC 38551; Petrie 1914, pl. 2:22f; Brunton 1928, pl. 95:16, 1948, pl. 31:33-34; PAHMA 6-10067 from N 570.

\section{N 2239 Map D4 (red)}

Green stone beads (PAHMA 6-14459). This object found with parts of wooden sandals, painted white, with remains of rush bindings. Round and square [cylindrical beads].

(1) PAHMA 6-14459 Loose beads

Exactly 2 beads.

Typology: Cylindrical, short—L16n; square-L17c. Material: Steatite.

Color: Green, brown, white. Length: 10.34-13.76 mm. Diameter: 5.27-5.43 mm. Aperture: $2.09-2.45 \mathrm{~mm}$. Weight: $1.2 \mathrm{~g}$.

No edge (cylindrical bead); edge (square bead). Aperture: End.

Perforation: Single plain.

Striations: Longitudinal (cylindrical); diagonal (square). Glaze: Mostly worn. 


\section{N 2291 Map C4}

Coptic grave. By head, one green glass bead (PAHMA 6-14550).

(1) PAHMA 6-14550 Loose bead

Exactly 1 bead.

Typology: Cylindrical, short-H6f. Material: Green feldspar.

Color: Green. Length: $3.53 \mathrm{~mm}$. Diameter: $4.27 \mathrm{~mm}$. Aperture: $1.25 \mathrm{~mm}$.

Weight: N/A (does not register). No edge.

Aperture: End. Perforation: Single plain. Striations: Rotary.

Polish: High.

\section{N 2292 Map C4; photo B 8251}

Scattered several glass beads [cylindrical and conical beads], and disc (PAHMA 6-14463, PAHMA 6-14464), 1 carnelian [biconical?] (PAHMA 6-14465). These objects were found with a bronze fragment (PAH MA 6-14465).

(1) PAHMA 6-14463 Length of necklace (not a loop) Approximately 18o beads. Typology: Disc, cylindrical- $\mathrm{R}_{52} \mathrm{f}$ (ostrich eggshell); ring, barrel-PN2c (faience). Material: Faience (approx. 178); ostrich eggshell (2).

Color: Blue, brown (faience); white (faience, ostrich eggshell). Length of strand: $24 \mathrm{~cm}$.

Length of beads: $0.64-2.62 \mathrm{~mm}$. Diameter of beads: $2.72-4.57 \mathrm{~mm}$. Weight: $2.6 \mathrm{~g}$. Production: омmA (faience). No edge.

Aperture: End.

Perforation: Single plain (ostrich eggshell). Glaze: Mostly worn (faience).

(2) PAH MA 6-14464 Length of necklace (not a loop) Exactly 28 beads.

Typology: Conical, short—PN24d (27); cylindrical, short—PDgl (1). Material: Faience.

Color: Blue, brown. Length of strand: $22.2 \mathrm{~cm}$.

Length of beads: $6.40-11.06 \mathrm{~mm}$. Diameter of beads: $3.58-5.17 \mathrm{~mm}$. Weight: $3.8 \mathrm{~g}$. Production: om MA (faience). No edge.

Aperture: End.

Decoration: Gadrooned (some conical beads); molded spiral decoration (cylindrical bead). Glaze: Mostly worn.

(3) PAHMA 6-14465 Loose beads, a broken pendant, and some fragments of metal (bronze?)

Exactly 8 objects ( 7 beads and 1 pendant). 
Typology: Barrel, short-PN16c (faience, 3); cylindrical, short-PN22e (faience, 1), L16l (glazed steatite, 2); a disc, biconical- $\mathrm{H}_{5} \mathrm{l}$ (carnelian, 1). Could not be determined: broken pendant (1). Material: Faience ( 4 beads, 1 pendant); carnelian (1); steatite (2).

Color: Red (carnelian), green (faience), white (faience and steatite). Length of objects: $2.07-5.64 \mathrm{~mm}$.

Diameter of objects: $2.00-8.46 \mathrm{~mm}$. Aperture of objects: $0.96-2.35 \mathrm{~mm}$. Weight: $0.7 \mathrm{~g}$.

Production: омма (faience); could not be determined (faience pendant). Edge (carnelian); no edge (the remainder).

Aperture: End.

Perforation: Double cone (carnelian), single plain (glazed steatite). Striations: Longitudinal (glazed steatite), random (carnelian).

Glaze: Mostly worn (faience, glazed steatite), mostly not worn (1 green faience, broken pendant). Polish: Dull (carnelian).

N 2432 Map D3; photo B 8251

Few black and blue glaze disc beads, large size (PAHMA 6-14471). These objects were found with the remains of a coffin, a piece of a nabut, and several rough sticks.

(1) PAHMA 6-14471 Loose beads

Exactly 36 beads.

Typology: Disc, barrel—PN2g, PN2j, PN2k. Material: Faience.

Color: Green, blue, black. Length: $1.28-2.77 \mathrm{~mm}$. Diameter: $4.36-5.70 \mathrm{~mm}$. Aperture: $1.03^{-1.95} \mathrm{~mm}$. Weight: $2.4 \mathrm{~g}$.

Production: om MA (faience). No edge.

Aperture: End.

Glaze: Mostly not worn.

N 2434 Map E3

In radim above remains of burial, few black and blue glaze beads, 1 crumb bead (PAHMA 6-14472).

(1) PAHMA 6-14472 Loose beads

Exactly 60 beads.

Typology: Barrel, long-PD48d (faience with crumb decoration, 1); disc, barrel—PN2k (19); ring, barrel—PN2g (40).

Material: Faience.

Color: Green, blue, black. Length: 1.15-7.73 mm. Diameter: 3.82-6.30 mm. Aperture: $0.90-1.87 \mathrm{~mm}$. Weight: $2.7 \mathrm{~g}$. 
Production: omma (faience). No edge.

Aperture: End.

Decoration: 1 faience has crumb decoration. Glaze: Mostly not worn.

The barrel bead has large chunks of glaze adhering to the surface. For a parallel, see PAHMA 6-14364 (N 2O71), UC 51425.

N 2521 Map N/A [ed.: This record is Reisner's note of preliminary work that was done in 1901.]

Alabaster beads in ghadim of irregular hole. Also, glazed stone and carnelian [ring/disc, barrel, and cylindrical beads] (PAHMA 6-12503). Also in ghadim were found turtle bones and pots: [jars] thrown ware, smoothed, painted red. [ed.: According to Hearst Museum records, PAHMA 6-12503 (beads) is from this tomb.]

(1) PAHMA 6-12503 Necklace

Approximately 426 beads.

Typology: Disc, barrel—PN2f (faience, approx. 413); disc, biconical- $\mathrm{H}_{5} \mathrm{i}$ (carnelian, 3); disc, cylindrical—H6r (carnelian, 9); teardrop—L15c (glazed steatite, 1 ).

Material: Faience (approx. 413), carnelian (12), steatite (1).

Color: Red, orange (carnelian); green, blue (faience); light brown (glazed steatite). Length of strand: $30.4 \mathrm{~cm}$.

Diameter of strand: $19.7 \mathrm{~cm}$. Length of beads: $0.61-7.17 \mathrm{~mm}$. Diameter of beads: 4.16-6.94 mm. Weight: $10.7 \mathrm{~g}$.

Production: оммв (faience).

Edge: 1 (glazed steatite), 3 (carnelian). Aperture: End.

Perforation type: Double cone (carnelian); plain (glazed steatite). Striations: Random (carnelian); longitudinal (steatite).

Polish: Moderate (carnelian).

Glaze: Mostly worn (faience, steatite).

N 2618 Map D5; photos C 8193, C 8520, C 8545, C 8594 (Fig. 7.1)

In large chamber, small gold bird amulet (PAHMA 6-14547). This object found with 12 pots all of one type in space $A$ and another in front of chamber.

(1) PAHMA 6-14547 Loose pendant

Exactly 1 pendant.

Typology: Figural—ibis on standard. Material: Gold.

Color: Silver, gold. 
Length of pendant (chest to back): $13.80 \mathrm{~mm}$. Diameter of pendant (head to standard): $22.13 \mathrm{~mm}$. Aperture: $1.22 \mathrm{~mm}$.

Weight: 2.0 g.

Production: Casting (metal).

For parallels, see Petrie 1914, pl. 42:247, BM EA 6246o.

N 2834 Map D3; photo C 8193 (Fig. 7.1)

In radim, 2 tiny glaze amulets (PAHMA 6-12515). In burial, badly broken up inscribed coffin in black paint.

(1) PAHMA 6-12515 Loose pendants

Exactly 2 pendants.

Typology: Figural—ankh sign (1), lion (1) Material: Faience.

Color: Blue.

Length: 1.48-2.65 mm. Diameter: 3.00-3.61 mm.

Aperture: N/A (too small to measure). Weight: N/A (did not register). Production: Molding (faience). Aperture: End.

Glaze: Mostly not worn.

For parallels to the lion, see Brunton 1928, pl. 95:15F6, ${ }_{15} \mathrm{Fg}$.

\section{Bibliography}

Andrews, C. 1997. Ancient Egyptian Jewelry. New York.

Beck, H. C. 2006. "Classification and Nomenclature of Beads and Pendants." BEADS: Journal of the Society of Bead Researchers 18:1-76. Available at: http://surface.syr .edu/beads/volı8/issi/4.

Brunton, G. 1948. Matmar. British Museum Expedition to Middle Egypt 1929-1931. London.

Germond, P. 2005. The Symbolic World of Egyptian Amulets, from the Jacques-Édouard Berger Collection. Milan.

Harrell, J. A. 2017. "A Preliminary Overview of Ancient Egyptian Stone Beads." Palarch's Journal of Archaeology of Egypt/Egyptology 14(2) (tEBP Series): 1-16.

Petrie, W. M. F. 1914. Amulets. Illustrated by the Egyptian Collection in University College, London. London.

Reisner, G. A. 1907. Amulets. CGC [35]. Cairo.

Reisner, G. A. 1908. Early Dynastic Cemeteries of Naga-ed-Dêr, Part I. University of California Publications, Egyptian Archaeology, Volume 2. [Leipzig]. 
Reisner, G. A. 1923. Excavations at Kerma, Parts $I V-V$. Harvard African Studies 6. Cambridge, MA.

Reisner, G. A. 1932. A Provincial Cemetery of the Pyramid Age, Naga-ed-Dêr Part III. University of California Publications, Egyptian Archaeology 6. Berkeley. Xia, N. 2014. Ancient Egyptian Beads. Beijing. DoI: 10.1007/978-3-642-54868-o. 